\title{
PRIMARY LARYNGEAL CANDIDIASIS WITH TUBERCULOSIS MIMICKING LARYNGEAL NEOPLASM
}

Aditya Prakash Misra. Hari Om Sharan, Ritu Mishra.

1. Associate Professor, Department of Radiodiagnosis, Rama medical College, Hospital and research centre, Kanpur, UP.

2. Assistant Professor, Department of Microbiology, Rama medical College, Hospital and research centre, Kanpur, UP.

3. Assistant Professor, Department of OBG, Rama medical College, Hospital and research centre, Kanpur, UP.

\section{CORRESPONDING AUTHOR}

Dr. Hariom Sharan

C/O, Aditya Prakash Misra, 2A/ 43, Azad Nagar, Kanpur, Uttar Pradesh. 208002.

E-mail: homsharan@gmail.com, saggimishra@rediffmail.com

Ph: . 00918005384934,00919839188620

ABSTRACT: A 50 -year-old man presented with hoarseness of voice, cough with expectoration for one month and severe dyspnoea from 15 days. C T Scan of the neck revealed soft tissue lesion circumferentially involving supra-glottic region with effacement of bilateral pyriform fossa predominantly on left side. Extralaryngeal spread to adjacent paralaryngeal space was also seen on left side. Marked airway luminal compromise was seen at the level of false vocal cord . Primary diagnosis of laryngeal malignancy was considered on findings revealed by CT scan neck. Direct laryngoscopy revealed erythema and ulcer with white pus discharge on left false vocal cord. A swab was taken from ulcer on left false vocal cord and sent for microbiological examination. The microbiological findings confirmed Mycobacterium tuberculosis with Candida albicans.. CONCLUSION: Primary candidiasis with tuberculosis in the laryngeal mucosa is a rare entity. The clinical and pathological presentations of laryngeal candidiasis and tuberculosis might be confused with those for malignant lesions if extralaryngeal spread is seen. Potential pitfalls in diagnosis and the importance of microbiological examination in rare case of dual superimposed primary laryngeal infections has been outlined.

KEYWORDS: Primary Laryngeal Candidiasis, Tuberculosis, mimicking, Laryngeal malignancy

INTRODUCTION: Primary candidiasis with tuberculosis in the laryngeal mucosa is a rare entity. The clinical and pathological presentations of laryngeal candidiasis and tuberculosis might be confused with those for benign or malignant lesions. We are presenting a case in which primary diagnosis of laryngeal malignancy was considered by findings revealed on CT scan neck but laryngoscopic and microbiological evaluation revealed and confirmed laryngeal infection i.e Primary Laryngeal Candidiasis with Tuberculosis. We have outlined potential pitfalls in diagnosis, and highlight the importance of evaluating microbiologically in rare case of dual superimposed primary laryngeal infections.

CASE REPORT: A 50 -year-old man presented with hoarseness of voice, cough with expectoration for one month and severe dyspnoea from 15 days. The patient has medical 
history of hypertension. Physical examination was otherwise unremarkable. Chest x-ray showed no evidence of pulmonary nodules, consolidation, or infiltrates.

Soft tissue computed tomography of the neck revealed soft tissue lesion circumferentially involving supra-glottic region with effacement of bilateral pyriform fossa predominantly on left side. Extralaryngeal spread to adjacent paralaryngeal space was also seen on left side(Fig1). Marked airway luminal compromise was seen at the level of false vocal cord (Fig 2). Few sub centimeter lymph nodes were seen in bilateral submandibular region.

Direct laryngoscopy revealed erythema and ulcer with white pus discharge on left false vocal cord(Fig 3). Swab was taken from ulcer on left false vocal cord and sent for microbiological culture and sensitivity. On Gram staining, Gram positive budding Yeast cells of Candida albicans were noted. On Ziehl Neelsen staining red coloured acid fast bacilli against blue background with budding yeast cells were seen with 20\% Sulphuric acid (Fig 4). These microbiological findings confirmed Mycobacterium tuberculosis with Candida albicans.

Patient received a course of 6 weeks fluconazole antifungal with 6 months of antitubercular therapy. Hoarseness resolved, and there has been no evidence of recurrence during the subsequent 24 months.

DISCUSSION: Primary laryngeal candidiasis along with tuberculosis is rare entity and can seldom be diagnosed clinically or radiologically. However, infective differential diagnosis may be kept if diffuse submucosal involvement is seen on soft tissue CT scan. Mycotic infections of the larynx are frequently seen in patients with immune insufficiency although they have also been reported in individuals with normal immune status ${ }^{1,2,3}$. Candidiasis is frequently seen in patients with immune deficiency syndromes or diabetes mellitus or those receiving immunosuppressive treatment ${ }^{3,4}$ and in individuals whose mucosal barriers are affected by excessive use of antibiotic or inhaled corticosteroids ${ }^{5,6}$ radiation therapy, smoking, trauma, gastro esophageal reflux or chemical-thermal damage ${ }^{4}$. Laryngeal candidiasis can present with various symptoms, such as odynophagia, dysphagia, hoarseness, respiratory distress and stridor 7,8 .Similarly laryngeal tuberculosis is almost always associated with pulmonary tuberculosis $9,10,11$.

In our patient soft tissue computed tomography of the neck showed soft tissue lesion circumferentially involving supra-glottic region. Effacement of bilateral pyriform fossa with predominant involvement on left side was noticed. Extralaryngeal spread to adjacent paralaryngeal space was seen on left side. Marked airway luminal compromise was seen. Laryngeal malignancy was considered as primary diagnosis as per findings.

The radiological findings of laryngeal tuberculosis depend on the stage and extension of lesion .In the infiltrative stage, there is focal thickening. In the ulcerative stage, the ulceration is not deep and rarely reaches the paraglottic spaces and the cartilage. Perichondritis is sometimes noted (epiglottis, arytenoids), but calcifications are not common and the paralaryngeal fat spaces are usually spared. The last stage is characterized by sclerosis. Various radiological findings that have been described include edema alone, an ulcero-infiltrative mass, infiltrative and pseudo-tumoral appearance (66\%); sub-glottic laryngitis (isolated swelling of the aryepiglottic fold or even massive cartilaginous ulceration and, sometimes, chondritis or perichondritis); diffuse form; and tuberculoma (enormous ventricular vegetation with a large base that elevates the ventricular strip) ${ }^{12,13}$.

Journal of Evolution of Medical and Dental Sciences/Volume1/Issue5/November-2012Page-849 
Previous studies showed that sparing of paralaryngeal spaces were suggestive of infective etiology ${ }^{14,15}$. However, in our case despite of infectious etiology paralaryngeal involvement was seen .

Laryngeal erythema and ulcer with white pus discharge on left false vocal cord was seen in our patient on laryngoscopy. The laryngoscopic appearance of candidiasis and tuberculosis generally includes oedema, erythema, ulceration, white plaque and pseudomembranous formations 5 .

SUMMARY: Characteristic CT findings of laryngeal tuberculosis include bilateral involvement, thickening of the free margin of the epiglottis, and extensive mucosal involvement. By comparison, laryngeal carcinoma present as unilateral involvement, infiltration of the pre epiglottic and paralaryngeal fat spaces by a submucosal mass, cartilage destruction, and extralaryngeal invasion. So due to paralaryngeal fat involvement malignancy was considered primary diagnosis in our case. Further evaluation is must before reaching to final diagnosis and starting treatment. In our case provisional diagnosis of malignancy was made as per radiological findings but confirmatory diagnosis was made after microbiological evaluation and patient was treated accordingly.

Conflicting Interests: The authors have no conflicting interests.

\section{REFERENCES:}

1. Makitie AA, Back L, Aaltonen LM, et al: Fungal infection of the epiglottis simulating a clinical malignancy. Arch Otolaryngol Head Neck Surg 2003;129: $124-126$.

2. Pabuççuog lu U, Tuncer C, Sengiz S: Histo-Pathology of candidal hyperplastic lesions of the larynx. Pathol Res Pract 2002; 198: 675 - 678.

3. Haberman RS, Becker ME, Ford CN: Candida epiglottitis. Arch Otolaryngol 1983; 109: $770-771$.

4. Forrest LA, Weed H: Candida laryngitis appearing as leukoplakia and GERD. J Voice 1998; 12: 91 - 95.

5. DelGaudio JM: Steroid inhaler laryngitis: Dysphonia caused by inhaled fluticasone therapy. Arch Otolaryngol Head Neck Surg 2002;128: 677 - 681.

6. Gallivan GJ, Gallivan KH, Gallivan HK: Inhaled corticosteroids: Hazardous effects on voice-An update. J Voice 2007; 21: 101 - 111.

7. Tashjian LS, Peacock JE: Laryngeal candidiasis. Arch Otolaryngol 1984; 110: 806 $-809$.

8. Ganesan S, Harar RP, Dawkins RS, et al: Invasive laryngeal candidiasis: A cause of stridor in the previously irradiated patient. J Laryngol Otol 1998; 112: 575 - 578.

9. Sá LC, Meirelles RC, Atherino CC, Fernandes JR, Ferraz FR: Laryngo-pharyngeal Tuberculosis. Braz J Otorhinolaryngol 2007;73:862-866.

10. Kozakiewicz J, Dec M, Gorczyca-Tarnowska J: The rare case of primary isolated tuberculosis in a 19 year-old patient. Otolaryngol Pol. 2006;60: 607-709.

11. Loehrl TA, Smith TL: Inflammatory and granulomatous lesions of the larynx and pharynx. Am J Med. 2001;111:113-117.

12. Aspestrand F, Kolbenstvedt A, Boysen M: CT findings in benign expansions of the larynx. J Comput Assist Tomogr. 1989;13:222-225. 
13. Jan A: Primary laryngeal tuberculosis (a case report) J Laryngol Otol. 1986;100:605-606.

14. Kim MD, Kim DI, Yune HY, Lee BH, Sung KJ, Chung TS, Kim SY: CT Findings of Laryngeal Tuberculosis: Comparison to Laryngeal Carcinoma. Journal of Computer Assisted Tomography. 1997; 21 : 29-34.

15. Jae-YL, Kwang-MK, Eun CC, Young-HK, Han SK ,Hong-SC: Current clinical propensity of laryngeal tuberculosis: review of 60 cases . European Archives of Oto-Rhino-Laryngology .2010;263:269.

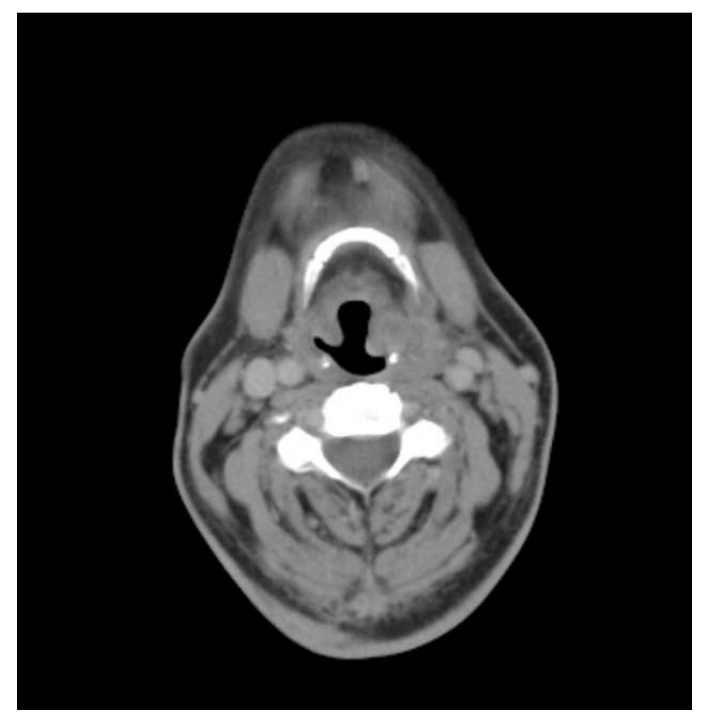

Figure 1: CT Scan neck shows soft tissue lesion circumferentially involving supra glottic region. Effacement of bilateral pyriform fossa with predominant involvement on left side noticed. Extralaryngeal spread to adjacent paralaryngeal space seen on left side.

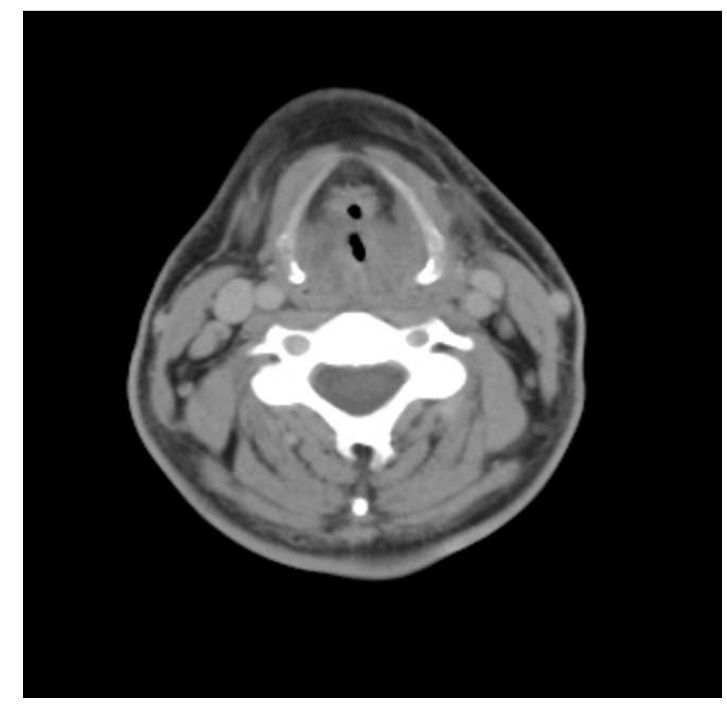

Figure 2: CT neck shows circumferential soft tissue infiltration at the level of false vocal cord. Marked airway luminal compromise also seen. 


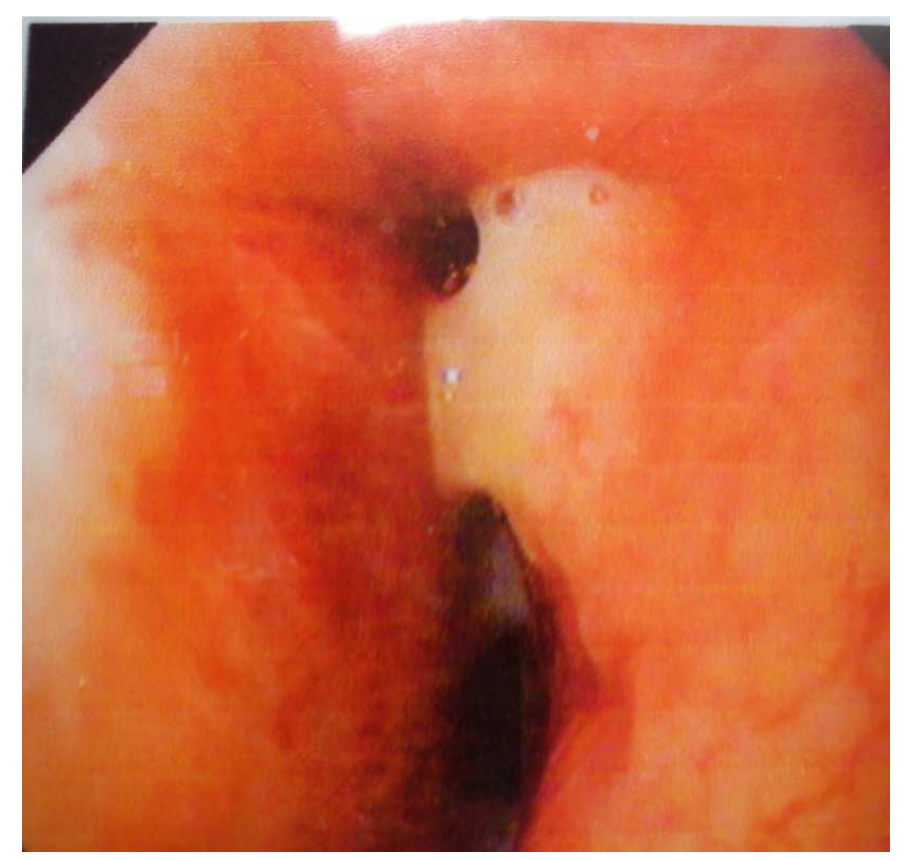

Figure 3: Direct laryngoscopy showing erythema and ulcer with white pus discharge on left false vocal cord.

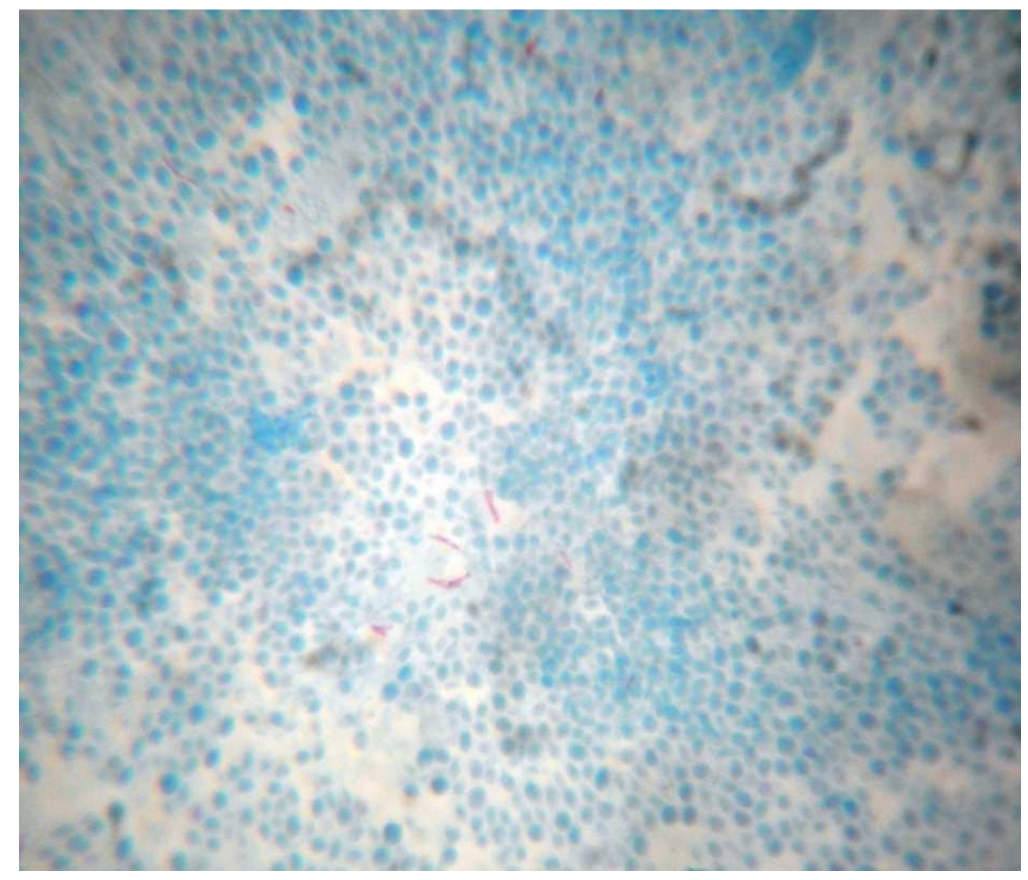

Figure 4: Ziehl Neelsen Staining (100x) : Acid Fast Bacilli with budding yeast cells. 